\title{
Computer-Controlled Test System for the Excitation of Very High Order Modes in Highly Oversized Waveguides
}

\author{
T. Ruess ${ }^{1, *}$, K. A. Avramidis ${ }^{1}$, G. Gantenbein ${ }^{1}$, Z. Ioannidis ${ }^{1}$, S. Illy ${ }^{1}$, F.-C. Lutz ${ }^{1}$, A. Marek ${ }^{1}$, S. Ruess ${ }^{1,2}$, \\ T. Rzesnicki ${ }^{1}$, M. Thumm ${ }^{1,2}$, D. Wagner ${ }^{3}$, J. Weggen ${ }^{1}$, and J. Jelonnek ${ }^{1,2}$ \\ ${ }^{1}$ IHM, ${ }^{2}$ IHE, Karlsruhe Institute of Technology (KIT), Kaiserstr. 12, D-76131 Karlsruhe, Germany \\ ${ }^{3}$ Max Planck Institute for Plasma Physics, Boltzmannstr. 2, D-85748 Garching, Germany
}

\begin{abstract}
The generation of a specific high order mode with excellent mode purity in a highly oversized cylindrical waveguide is mandatorily required for the verification of high power components at sub- $\mathrm{THz}$ frequencies. An example is the verification of quasi-optical mode conversion and output systems for fusion gyrotrons. A rotating high order mode can be excited by taking a low power RF source (e.g. RF network analyser) and by injecting the RF power via a horn antenna into a specific adjustable quasi-optical setup, the so-called mode generator. The manual adjustment of the mode generator is typically very time consuming. An automatized adjustment using intelligent algorithms can solve this problem. In the present work the intelligent algorithms consist of five different mode evaluation techniques to determine the azimuthal and radial mode indices, the quality factor, the scalar mode content and the amount of the counter-rotating mode. Here, the implemented algorithms, the design of the computer-controlled mechanical adjustment and test results are presented. The new system is benchmarked using an existing $\mathrm{TE}_{28,8}$ mode cavity operating at $140 \mathrm{GHz}$. In addition, the repeatability of the algorithms has been proven by measuring a newly designed $\mathrm{TE}_{28,10}$ mode generator cavity. Using the described advanced mode generator system, the quality of the excited modes has been significantly improved and the time for the proper adjustment has been reduced by at least a factor of 10 .
\end{abstract}

Keywords low power measurement; quasi-optical mode generator; high order modes; automated measurement setup; mode evaluation techniques; gyrotron; fusion plasmas

\section{Introduction}

Very high RF power can be propagated at sub-THz frequencies by using the $\mathrm{HE}_{11}$ mode in corrugated waveguides and the TEM $\mathrm{M}_{00}$ mode for optical transmission. These modes are low order modes having low transmission losses compared with the high order modes. In contrast, high order modes are used in oversized waveguides for the generation of high RF power (up to $2 \mathrm{MW}$ or even more) having a lower wall loading compared with the low order modes. Applications can be found e.g. in communication [1] and plasma heating for fusion plants [2]. In latter application, gyrotrons are the high power RF sources used for Electron Cyclotron Heating, Current Drive (EC H\&CD) and for stability control of the fusion plasma. Today, fusion gyrotrons can generate up to $2.2 \mathrm{MW}$ RF output power [3] in ms pulses. The proper verification of the oversized waveguide components and mode converters of those gyrotrons is vital to prevent catastrophic failures [4]. Their quasi-optical mode converter consists of the launcher and three mirrors. Those components convert the high order rotating waveguide mode, excited in the interaction cavity, into the linearly polarized fundamental Gaussian beam (TEM 00 mode). The low power $(\sim 1 \mathrm{~mW}$ - cold test) verification of this converter system is vital before final installation into the high power fusion gyrotron because a launcher does not allow any design failures, and manufacturing tolerances of only a few microns are acceptable for proper operation. With a low power test system, the verification of proper launcher operation is performed using a mode generator. Up to now, the fully manual adjustment of the mode generator can be a very time consuming task and does not always result in a high mode purity. The major issues are high spectral density of the mode spectrum in oversized waveguides, as well as the large number of degrees of freedom of the mode generator setup and the manually adjustable sliding units. Additionally, the existing measurement equipment cannot provide the required resolution and is limited to a frequency of $170 \mathrm{GHz}$, so is not adequate for operation at higher frequencies. Therefore, this paper describes an advanced mode generator setup using a new

* Corresponding author: tobias.ruess@ kit.edu 
VNA (Vector Network Analyser) and high-precision linear drivers for computer controlled adjustment. An algorithm has been implemented with the goal to reduce the time of the adjustment procedure by finding the correct mode in an automated way. In this algorithm, five mode evaluation techniques are implemented for the determination of the measured mode and its quality. An existing $\mathrm{TE}_{28,8}$ mode generator cavity has been used to benchmark the automated test setup and the algorithm.

\section{Advanced Measurement Setup}

A photograph of the mode generator setup is shown in Fig. 1, where the principle is based on a quasi-optical approach [5]. The RF signal, which is generated by the VNA has a constant frequency and is transmitted using a circular $\mathrm{HE}_{11}$ mode horn antenna. A lens system converts the transmitted spherical wave into an astigmatic Gaussian like beam (TEM 00 mode) having a plane phase front at the quasi-parabolic (q.-p.) mirror in order to minimise phase changes. The astigmatism is introduced in order to increase the efficiency of the mode generator by only illuminating the q.-p. mirror. Thereby spillover losses are limited. The q.-p. mirror focuses the incoming wave onto the caustic radius of the preferred mode inside the mode generator cavity. The profile of the cavity is specially designed for this application using a scattering matrix code [6]. The cavity contour is very similar to the one of the gyrotron cavity having a cut-off section at one side and an up-taper at the other side, thus forming an open resonator. As in coaxial-cavity gyrotrons, a metallic insert is used to assist the easier excitation of the desired mode $[7,8]$. The outer wall of the cavity is perforated in the midsection area [9], so that the wave beam can couple to the resonant mode in the cavity. The correct $\mathrm{TE}_{\mathrm{m}, \mathrm{n}}$ mode, where $m$ is the azimuthal and $n$ the radial index, is excited depending on the dimensions, the frequency and the irradiation of the midsection. The radial and azimuthal field components are given by:

$$
\begin{gathered}
E_{r}=-\frac{m}{r} \cdot J_{m}\left(\frac{\chi_{m, n} \cdot r}{R}\right) \cdot \exp \left(-1 \cdot i\left(m \cdot \varphi+k_{z} \cdot z\right)\right), \\
E_{\varphi}=1 i \cdot \frac{\chi_{m, n}}{R} \cdot J_{m}^{\prime}\left(\frac{\chi_{m, n} \cdot r}{R}\right) \cdot \exp \left(-1 \cdot i\left(m \cdot \varphi+k_{z} \cdot z\right)\right)
\end{gathered}
$$

where $R$ is the outer cavity radius, $\chi_{m, n}$ the eigenvalue and $k_{z}$ the wavenumber in propagation direction. An additional non-linear up-taper is mounted at the end of the cavity to increase the resolution of the measurement system. The field at the end of the taper is measured with a rectangular standard waveguide pick-up antenna. This antenna is mounted on a 3D measurement arm, where a stepwise 2D scan of the plane parallel to the output of the mode generator can be taken. In this setup the transmission coefficient $S_{21}$ is measured in amplitude and phase.

The existing measurement setup presented in [10] offers several issues: (i) the in-house manufactured network analyser cannot provide sufficient resolution and does not provide frequencies above $170 \mathrm{GHz}$, (ii) the mechanical sliding units for the q.-p. mirror show a relatively large amount of positioning hysteresis and (iii) the manual adjustment of the q.-p. mirror using the mechanical sliding units is very time consuming. For these reasons, the setup has been revised to achieve better results. The advanced schematic is shown in Fig. 2. The VNA covers the frequency range from $140-330 \mathrm{GHz}$ using extension modules. The mechanical sliding units are replaced by two high-precision linear drivers (tables) having a position error of $<25 \mu \mathrm{m} / 100 \mathrm{~mm}$ and a repetition failure (bidirectional) of $<15 \mu \mathrm{m}$. The setup allows a complete sequence of measurements during a long period of time using the computer-based adjustment of the q.-p. mirror and the VNA.

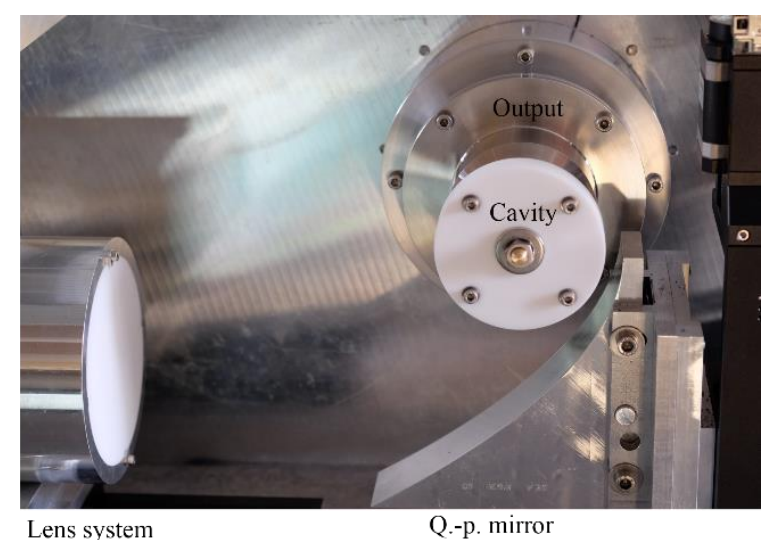

Fig. 1. Photo of the mode generator setup with two highprecision linear tables in $\mathrm{z}$ - and y-direction.

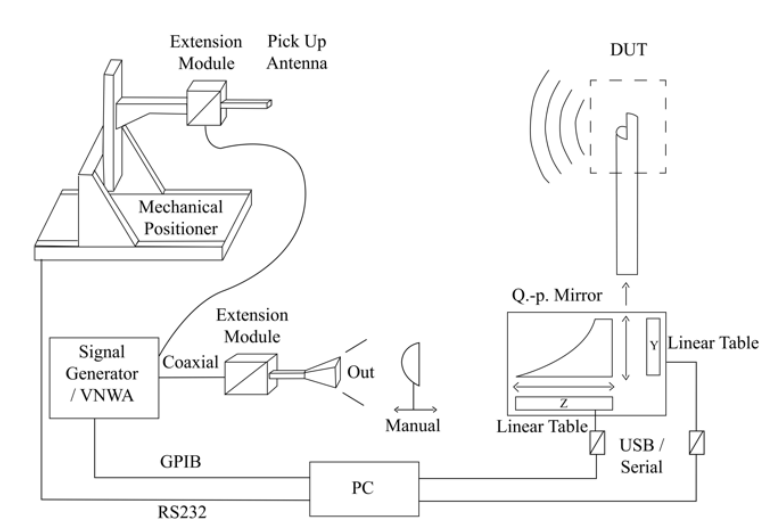

Fig. 2. Computer-based measurement setup using a vector network analyser and high-precision linear tables for the adjustment of the quasi-parabolic mirror in $\mathrm{z}$ - and $\mathrm{y}$-direction. 


\section{Intelligent Algorithms for Automatic Adjustment}

The most challenging and time-consuming task is given by the excitation of the correct mode. To improve this situation, the measurement procedure shown in Fig. 3 has been developed using an automated adjustment [11]. First of all, the transmitting horn antenna (Tx antenna), the lens system, the q.-p. mirror and the cavity is implemented as a 2D model with MATLAB. The ideal distances between antenna and lens system, and q.-p. mirror and cavity can be calculated and are used as a start position for the optimisation. In the next step of the procedure, called "Lens System Adjustment", the calculated position for the lens system is proven by measurements. This step cannot be done automatized because the lens system has to be disassembled from the setup. Using the ideal simulation values, the distances are checked between antenna and lens system, and between lens system and receiving $(\mathrm{Rx})$ antenna, which is a placeholder for the q.-p. mirror. Through this measurement, a wave with only a minimum phase change (plane wave) can be achieved at the plane where the q.-p. mirror is positioned.

In the next step, the lens system is assembled to the mode generator setup again and the operating frequency of the designed cavity has to be determined. The transmission coefficient is plotted in Fig. 4 for a frequency sweep from $138-142 \mathrm{GHz}$, where the Rx pick-up antenna is positioned on the field maximum of the desired mode. There are three main maxima which indicate the frequencies of excited modes using a certain q.-p. mirror position. These resonant frequencies are at 140.006, 138.07 and 140.85 GHz. The implemented algorithm searches for the amplitude peak which is closest to the nominal frequency (here $140.0 \mathrm{GHz}$ ) in the first iteration.

The closest peak at $140.006 \mathrm{GHz}$ is chosen for the next step "Find Correct Mirror Position". A change of the mirror position in an area of $\approx 5 \times 5 \mathrm{~mm}$ around the ideal q.-p. mirror position, which is calculated using the already mentioned MATLAB code, is performed where the Rx antenna is still positioned on the field maximum of the desired mode. The q.-p. mirror is driven with a step size of $0.1 \mathrm{~mm}$ in the before mentioned $5 \mathrm{x} 5 \mathrm{~mm}$ area in $\mathrm{x}$ - and y-direction. The result of this procedure step can be seen in Fig. 5. The graph shows the amplitude in dependence of the q.-p. mirror position. The mirror position with the highest amplitude value (here: $\mathrm{x}=69.2 \mathrm{~mm}, \mathrm{y}=85.2 \mathrm{~mm}$ ) represents an adjustment which excites a mode with a high quality. This position can differ from the ideal position due to fabrication tolerances and mirror rotation. A mode pattern with low resolution (pixel size: $0.5 \times 0.5 \mathrm{~mm}$ ) is taken with the chosen q.-p. mirror position. The acquired mode pattern is evaluated using the mode evaluation techniques presented in Ch. 4: Mode Evaluation Techniques where the excited $\mathrm{TE}_{m, n}$ mode can be determined. After a successful evaluation, the code can determine if the excited mode is the correct one or not and gives a statement about its purity. If the excited mode seems to be not correct, the procedure does the second iteration by

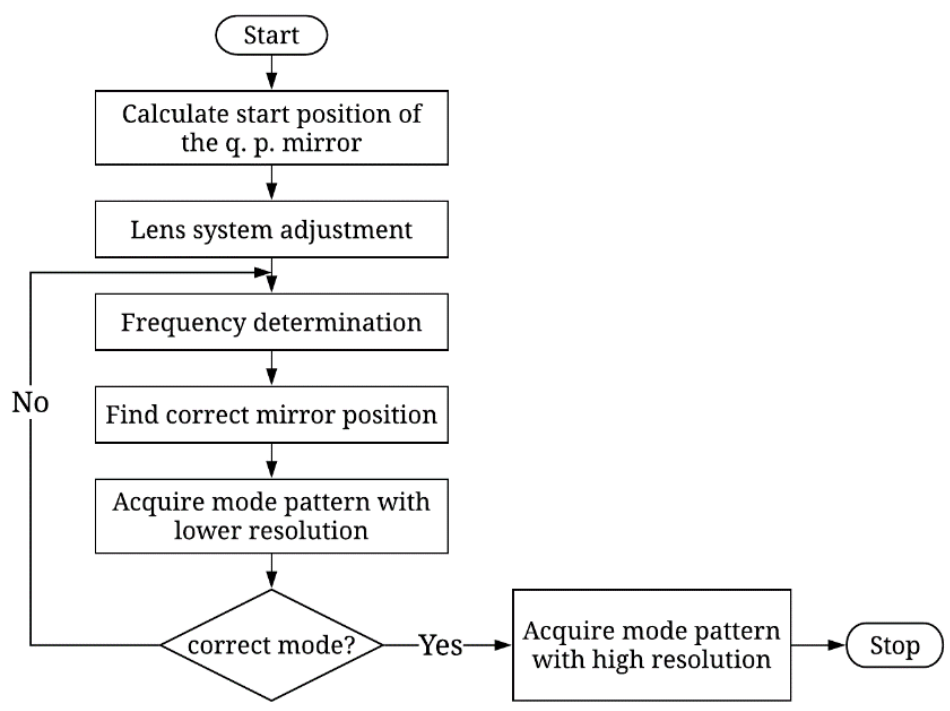

Fig. 3. Measurement procedure for a time saving mode evaluation algorithm.

searching the second peak in the frequency range from $138-142 \mathrm{GHz}$. But, if the correct mode is excited, the mode pattern is measured with higher accuracies (pixel size: $0.2 \times 0.2 \mathrm{~mm}$ ). In the following, fine tuning can be done. Therefore, mode patterns with high resolution are taken with a change of $\pm 0.1-0.2 \mathrm{~mm}$ in the $\mathrm{x}$ - and $\mathrm{y}$ direction of the q.-p. mirror to probably increase the purity of the excited mode. The launcher and the mirror system can be verified after the successful excitation of the mode using this procedure. 


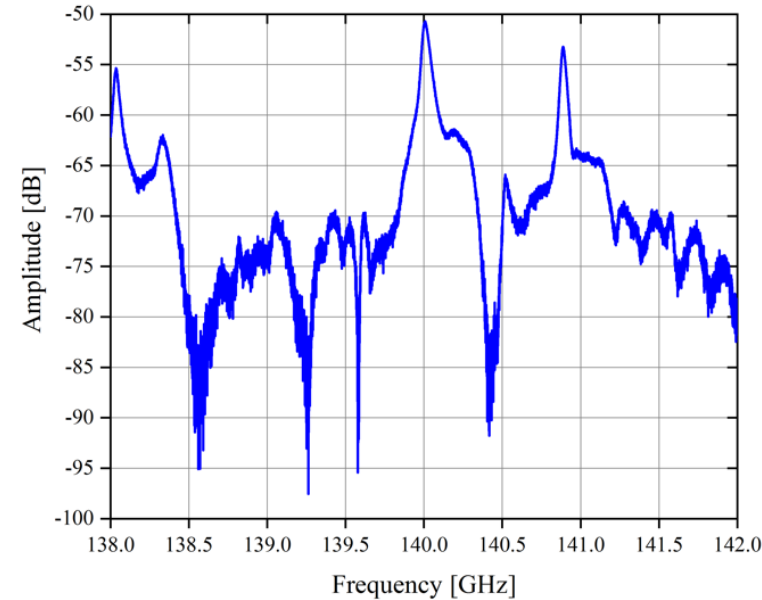

Fig. 4. Amplitude measurement in the frequency range from $138-142 \mathrm{GHz}$, whereby the Rx-antenna is placed on the field maximum of the desired mode.

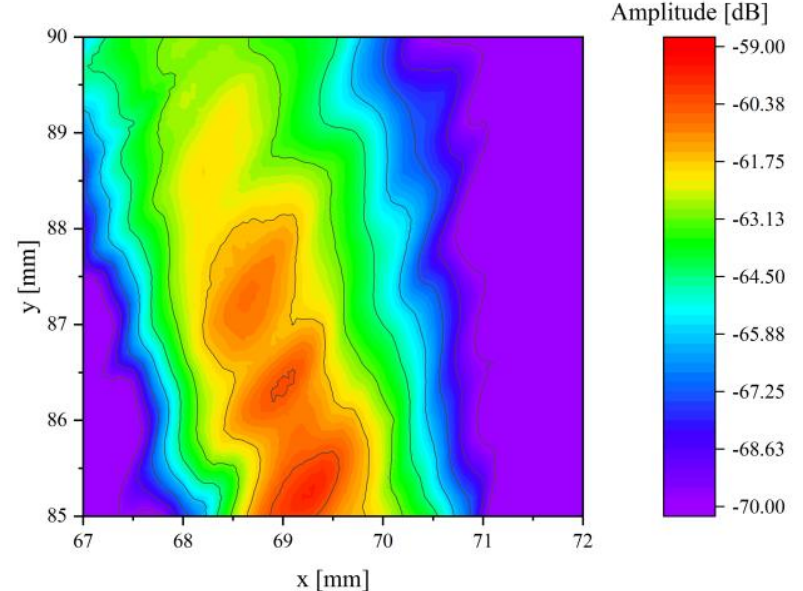

Fig. 5. Field amplitude in dependence of different q.-p. mirror positions, placing the Rx-antenna on the field maximum of the desired mode.

\section{Mode Evaluation of the Final Results of Adjustment}

A mode pattern with high resolution is depicted in Fig. 6. The Rx pick-up antenna is an open-ended standard waveguide where only the linear polarized fundamental $\mathrm{TE}_{10}$ mode can propagate. Due to the polarization of the antenna, the amplitude of the measured field components of the mode pattern depends on their orientation. Therefore, $E_{\mathrm{vertical}}\left(E_{\mathrm{y}}\right)$ and $E_{\text {horizontal }}\left(E_{\mathrm{x}}\right)$ are measured instead of $E_{r}$ and $E_{\varphi}$. The electric field components of the two different polarizations are obtained by a transformation from polar to Cartesian coordinates:

$$
\begin{aligned}
& E_{x}=E_{r} \cdot \cos (\varphi)-E_{\varphi} \cdot \sin (\varphi), \\
& E_{y}=E_{r} \cdot \sin (\varphi)+E_{\varphi} \cdot \cos (\varphi) .
\end{aligned}
$$

This measurement procedure of the mode pattern leads to a not fully depicted caustic. The reason for this effect is, that on the caustic radius the $E_{\varphi}$ component is equal to 0 and so, only the radial field component is present which leads to a very high amplitude in only one direction (vertical in Fig. 6) and very low in the orthogonal one (horizontal) where only the cross polarization is measured. Additionally, the image is blurred in the diagonally crosswise space, where both components (radial and azimuthal) are present. The second polarization can be measured by rotating the Rx pick-up antenna by 90 degrees, resulting in a measured mode pattern which is also rotated by 90 degrees. Any asymmetry in the setup can lead to the excitation of the counter-rotating mode which will cause some amount of standing wave which will appear as nodes in the mode pattern.

Five different evaluation techniques are implemented to determine the indices $m$ and $n$ of the $\mathrm{TE}_{m, n}$ mode and its quality. Firstly, the radial index $n$ can be determined by counting the number of rings. Figure 7 shows a cut view through the centre of the vertical field amplitude image. The measurement results are compared with the simulations and show a very good agreement. The two main peaks are allocated to the field maxima. The amplitude levels show a small discrepancy. The difference in the amplitude level is attributed to the rotation of the q.-p. mirror and so to the irradiation into the cavity. Further, the mode pattern is rotated due to the Rx antenna by $\approx 12^{\circ}$, while the simulated pattern is not rotated, which influences the evaluation and the peak amplitude, as well.

Secondly, the scalar mode content can be calculated and amounts to $94.5 \%$ in this case. This value is expected to be higher in reality because the mode pattern is not measured inside the waveguide due to the setup and so the mode pattern will diverge. Additionally, the mode is slightly rotated which influences the calculated result, as well.

Thirdly, the azimuthal index $m$ can be determined by evaluating the phase. The measured phase is shown in Fig. 8. In the centre of the pattern the phase is random because of the very small power of $E_{r}$ and $E_{\varphi}$. The algorithm evaluates the phase on the circumference of a circle with constant radius as shown in Fig. 8. Two decision criteria are implemented for a robust evaluation. These are: (i) counting the number of phase jumps (positive peaks) and (ii) counting the zero-crossings and divide the number by two. As already published in [12], the counted number of phase periods has to be added by one. This effect comes along that the azimuthal index $m$ is reduced by one if only one field component (horizontal or vertical) is measured. This assumption is proved starting with the trivial case where the amplitudes of $E_{r}$ and $E_{\varphi}$ are similar, assuming $a=b$ using $a=-\frac{m}{r} J_{m}\left(\frac{\chi}{R} \cdot r\right)$ and $b=\frac{\chi}{R}$. $J_{m}^{\prime}\left(\frac{\chi}{R} \cdot r\right)$. The z-position is fixed and could be neglected by the use of $z=0$. Exemplarily, only the $E_{y}$ component is derived using eq. (4):

$$
E_{y}=a \cdot \exp (-1 \mathrm{i} \cdot m \cdot \varphi) \cdot \sin (\varphi)+b \cdot \exp (-1 \mathrm{i} \cdot m \cdot \varphi) \cdot \cos (\varphi),
$$

writing the trigonometric terms as an exponential function: 


$$
\begin{gathered}
E_{y}=a \cdot \exp (-1 \mathrm{i} \cdot m \cdot \varphi) \cdot \frac{\exp (i \varphi)-\exp (-i \varphi)}{2}+a \cdot \exp (-1 \mathrm{i} \cdot m \cdot \varphi) \cdot \frac{\exp (i \varphi)+\exp (-i \varphi)}{2}, \\
E_{y}=a \cdot \exp (-1 \mathrm{i} \cdot \varphi(m-1)) .
\end{gathered}
$$

The argument $\arg \left(E_{y}\right)$ of eq. 5 is set to zero, whereby the number of zero crossings can be found.

$$
\begin{gathered}
\arg \left(E_{y}\right)=\frac{\sin (-\varphi(m-1))}{\cos (-\varphi(m-1))}=0 \\
\varphi=\frac{\pi \cdot n}{m-1},
\end{gathered}
$$

where $n \in \mathbb{Z}$. Equation 6 implies that the pattern has $2(m-1)$ zero-crossings or $m-1$ phase jumps over a period of $2 \pi$. The non-trivial case $(a \neq b)$ can be solved assuming $\frac{a}{m} \leq b \leq 5 a$ and $-5 a \leq b \leq-\frac{a}{m}$ for $a<0$, where eq. 6 is valid, as well. The evaluation on the circle is most robust if the evaluation radius is slightly smaller than the caustic radius. Figure 9 shows the 2D plot of the unwrapped phase on the circumference of the circle. The phase difference between the measured phase and a linear fitted one is evaluated. This phase difference shows a rotation of $\approx 12^{\circ}$, which can be seen in the amplitude mode pattern in Fig. 6, as well. The areas around $-102^{\circ}$ (ideally $-90^{\circ}$ ) and $+78^{\circ}$ (ideally $+90^{\circ}$ ) are highlighted and marks the section where only the $E_{r}$ component is measured. The phase difference is increased or respectively decreased in the greyed area. In this area the amplitude of the radial $E_{r}$ component is decreasing and the amplitude of the azimuthal $E_{\varphi}$ component is increasing. The phase change in this area is because of the mixture of $E_{r}$ and $E_{\varphi}$, which have a $90^{\circ}$ phase shift to each other, as presented in eq. (1) and (2).

The fourth mode evaluation technique concerns to the amount of the counter-rotating mode. The complete suppression of the counter-rotating mode is not possible due to the unsymmetrical principle of the excitation. The amount of the counter-rotating mode is an important number to verify the quality of the mode generator and is defined by the power ratio between the unwanted counter-rotating (-) mode and the wanted co-rotating (+) mode. The formula is given by [13]:

with

$$
\frac{p^{-}}{p^{+}}=\left(\frac{\Delta E-1}{\Delta E+1}\right)^{2}
$$

$$
\Delta E=10^{\frac{E_{\max } / \mathrm{dB}-E_{\min } / \mathrm{dB}}{20}},
$$

where $E_{\max }$ and $E_{\min }$ are described by the maximal and minimal measured field amplitudes along the first azimuthal ring in the mode pattern and $p^{+}$and $p^{-}$are related to the relative power fractions of the co- and counterrotating mode. The counter-rotating amount is calculated to be $0.33 \%$, which indicates a good cavity design and adjustment of the system. The algorithm used for the phase evaluation is applied with only small modifications in this evaluation method, as well.

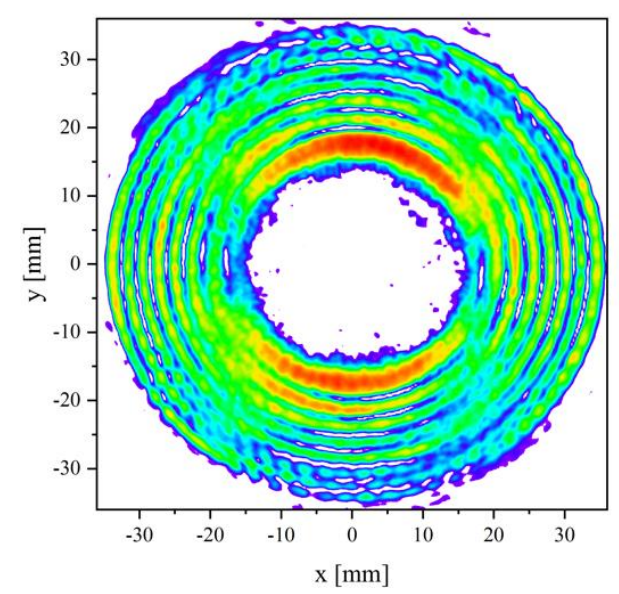

Fig. 6. Measured amplitude pattern of the $\mathrm{TE}_{28,8}$ mode operating at $140.006 \mathrm{GHz}$.
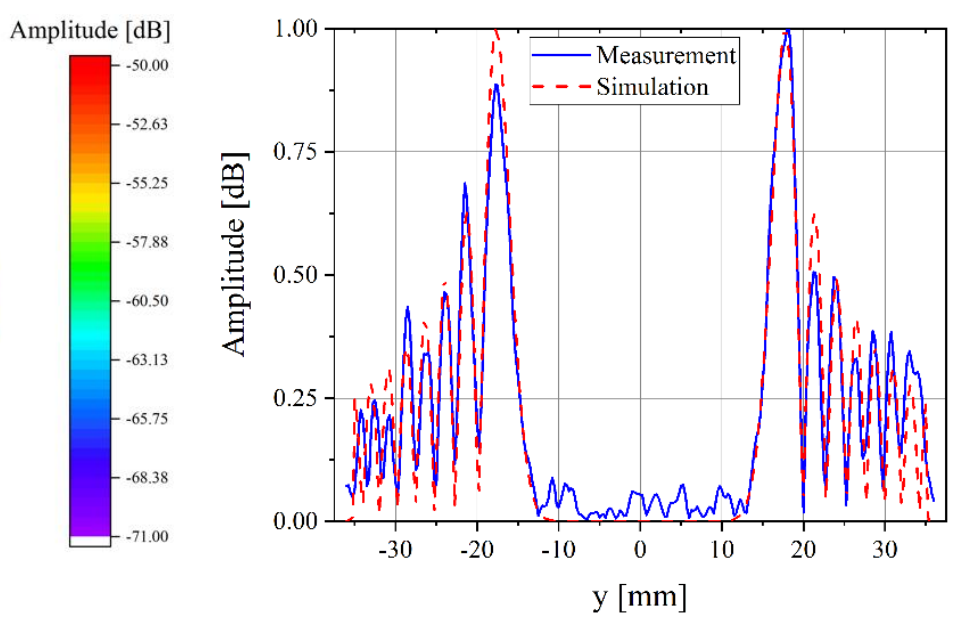

Fig. 7. Vertical cut of the amplitude mode picture from the $\mathrm{TE}_{28,8}$ mode measurement and simulation.

Fifthly, the measured quality factor of the cavity can be compared with the simulated one. The measured quality factor is determined from Fig. 4 by $Q=f_{\text {res }} / B_{3 \mathrm{~dB}}=2548$ and is in good agreement with the designed cavity quality factor of 2533 . 


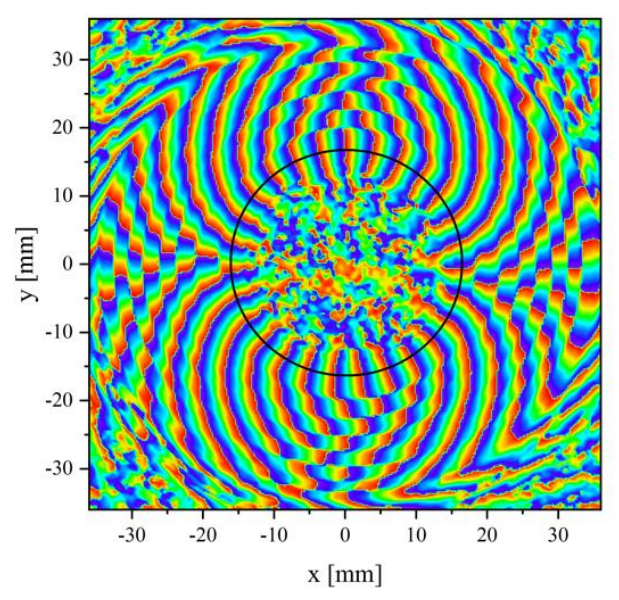

Fig. 8. Measured phase of the $\mathrm{TE}_{28,8}$ mode operating at $140.006 \mathrm{GHz}$.

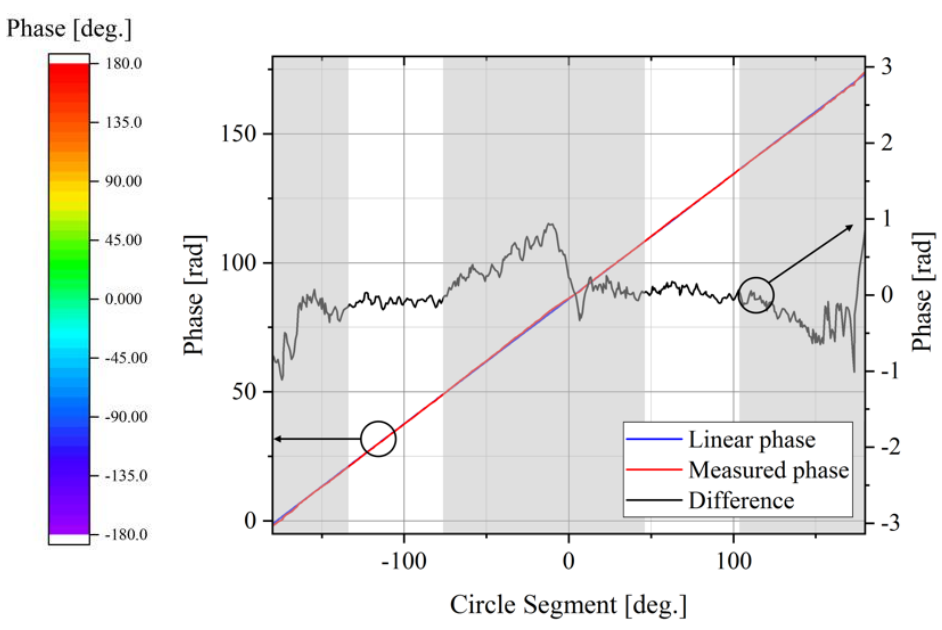

Fig. 9. Unwrapped phase from the measured $\mathrm{TE}_{28,8}$ mode pattern on the circumference of a constant radius compared with a linear fitted phase. The difference phase is calculated by subtracting the measured and linear phase.

\section{Repeatability Test for $\mathbf{T E}_{28,10}$ mode generator cavity}

For a 1.5 MW gyrotron which is under discussion for upgrading the existing $1 \mathrm{MW} \mathrm{TE}_{28,8}$ mode gyrotrons for W7$\mathrm{X}$ (Wendelstein 7-X [14]) the $\mathrm{TE}_{28,10}$ mode operating at $140 \mathrm{GHz}$ has been selected as a suitable cavity mode [15]. A $\mathrm{TE}_{28,10}$ mode generator cavity has been fabricated and tested using both mode generator test setups. In this study a time saving of a factor 10 and additionally higher mode purity has been achieved. In Figs. 10 and 11 the measured amplitude and phase pattern are plotted. The measured mode is confirmed to be the $\mathrm{TE}_{28,10}$ mode operating at $140.01 \mathrm{GHz}$ using the given mode evaluation techniques. The algorithms calculated that the scalar mode content is $93 \%$, the counter-rotating amount is $0.57 \%$ and the quality factor is 2522 , which is in good agreement with the simulated quality factor of 2506 .

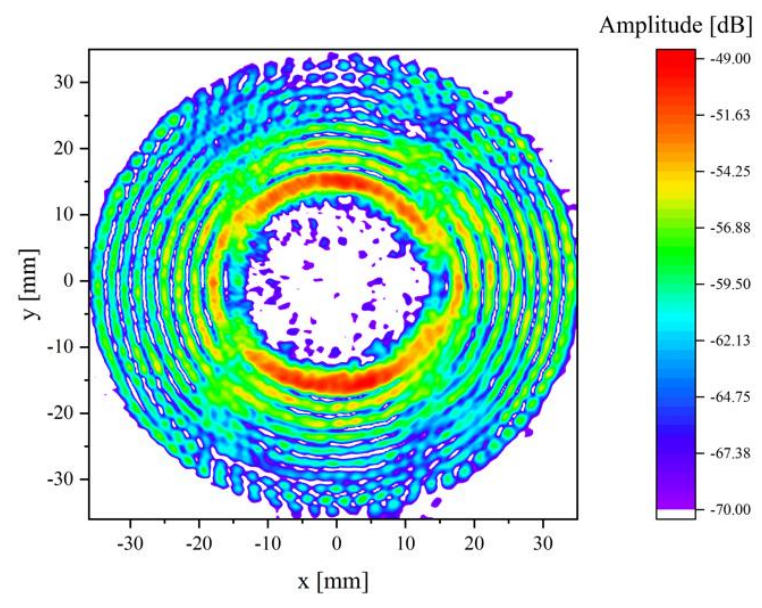

Fig. 10. Measured amplitude pattern of the $\mathrm{TE}_{28,10}$ mode operating at $140.006 \mathrm{GHz}$.

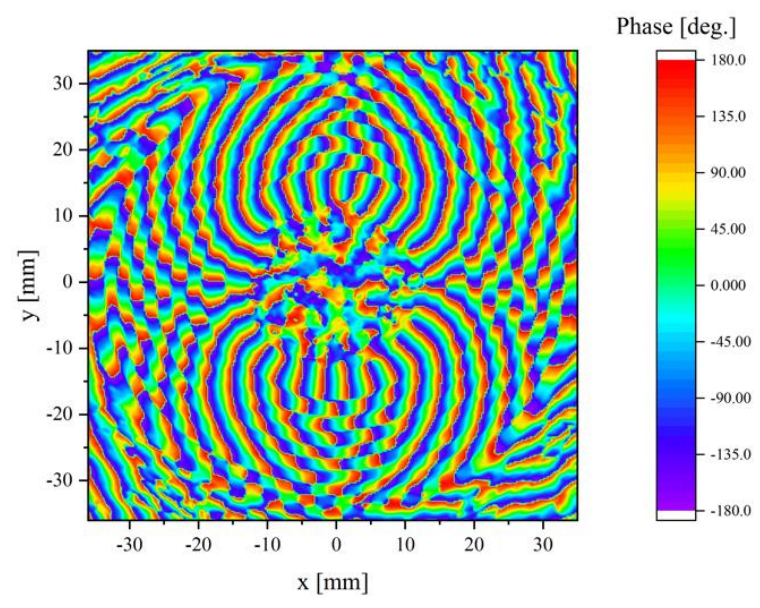

Fig. 11. Measured phase pattern of the $\mathrm{TE}_{28,10}$ mode operating at $140.006 \mathrm{GHz}$.

\section{Summary and Outlook}

In this paper, the automatized adjustment of a gyrotron mode generator for cold tests is shown to provide the excitation of very high order rotating modes in waveguides with a high mode purity. The old test setup has been modified installing a VNA, high precision linear drivers and 3D measurement arm which are controlled via computer. Therefore, higher position and measurement accuracies are achieved. The computer-based controlling allows a positioning which is independent of human attendance. Five mode evaluation techniques are implemented determining the azimuthal and radial mode index, the scalar mode content, the amount of the counter-rotating mode and the quality factor. The algorithm is able to efficiently detect, whether the measured mode pattern matches with the given theoretical mode or not. The newly designed and presented measurement system is benchmarked 
using an existing $\mathrm{TE}_{28,8}$ mode cavity operating at $140.006 \mathrm{GHz}$ achieving a mode purity of approximately $95 \%$ and a counter-rotating amount of only around $0.4 \%$. The repeatability of the procedure was successfully demonstrated for a $\mathrm{TE}_{28,10}$ mode cavity, where an adjustment time saving of a factor of approximately 10 was achieved.

\section{References}

1. A. Sawant, et al., Scientific Reports, vol. 7, no.: 3372 (2017)

2. T. Omori, et al., Fusion Eng. Des., vol. 86, pp. 951-954, 2011.

3. T. Rzesnicki, et al., Trans. On Plasma Science, vol. 38, no. 6, pp. 1141-1149, 2010.

4. T. Ruess, et al., EPJ Web Conf., vol. 187 (2018).

5. N. Aleksandrov, et al., Int. J. Infrared and Millimeter Waves, 13, 1369-1385, 1992.

6. D. Wagner, et al., Int. J. Infrared and Millimeter Waves 19,185-194 (1998).

7. C. T. Iatrou, et al., IEEE Trans. Microwave Theory Tech., vol. 44, pp. 56-64, 1996.

8. K. A. Avramides, et al., IEEE Trans. on Plasma Science, vol. 32, no. 3, 2004.

9. M. Pereyaslavets, et al., Int. J. Electronics, 82:1, pp. 107-115, 1997.

10. M. Losert, J. Jin and T. Rzesnicki, in IEEE Trans. on Plasma Science, vol. 41, no. 3, pp. 628-632, March 2013.

11. T. Ruess, et al., EPJ Web Conf., (2018).

12. M. Losert, et al., 2015 German Microwave Conference, Nuernberg, 2015, pp. 256-259.

13. A. Arnold, G. Dammertz and M. Thumm, IRMMW, Conference Digest of the 2004 Joint 29th International Conference on 2004 and 12th International Conference on Terahertz Electronics, 2004., 2004, pp. 671-672.

14. H. Braune, et al, in Proc. IRMMW, Copenhagen, Denmark, Sep. 2016, pp. 1-2.

15. K. A. Avramidis, et al., 20th Joint Workshop on Electron Cyclotron Emission (ECE) and Electron Cyclotron Resonance Heating (ECRH), May 2018, Greifswald, Germany. 